\title{
QUEEN'S
UNIVERSITY
BELFAST
}

\section{Continuous statistical modelling in characterisation of complex hydrocolloid mixtures using near infrared spectroscopy}

Georgouli, K., Carrasco, B., Vincke, D., Martinez del Rincon, J., Koidis, A., Baeten, V., \& Fernández Pierna, J. A. (2020). Continuous statistical modelling in characterisation of complex hydrocolloid mixtures using near infrared spectroscopy. Chemometrics and Intelligent Laboratory Systems, 196, [103910]. https://doi.org/10.1016/j.chemolab.2019.103910

Published in:

Chemometrics and Intelligent Laboratory Systems

Document Version:

Peer reviewed version

Queen's University Belfast - Research Portal:

Link to publication record in Queen's University Belfast Research Portal

\section{Publisher rights}

Copyright 2019 Elsevier.

This manuscript is distributed under a Creative Commons Attribution-NonCommercial-NoDerivs License

(https://creativecommons.org/licenses/by-nc-nd/4.0/), which permits distribution and reproduction for non-commercial purposes, provided the author and source are cited.

\section{General rights}

Copyright for the publications made accessible via the Queen's University Belfast Research Portal is retained by the author(s) and / or other copyright owners and it is a condition of accessing these publications that users recognise and abide by the legal requirements associated with these rights.

Take down policy

The Research Portal is Queen's institutional repository that provides access to Queen's research output. Every effort has been made to ensure that content in the Research Portal does not infringe any person's rights, or applicable UK laws. If you discover content in the Research Portal that you believe breaches copyright or violates any law, please contact openaccess@qub.ac.uk. 


\title{
Continuous statistical modelling in characterisation of complex hydrocolloid mixtures using near infrared spectroscopy
}

\author{
Konstantia Georgouli $^{\mathrm{a}, *}$, Beatriz Carrasco ${ }^{\mathrm{b}}$, Damien Vincke ${ }^{\mathrm{c}}$, Jesus Martinez \\ Del Rincon ${ }^{\mathrm{d}}$, Anastasios Koidis ${ }^{\mathrm{a}, *}$, Vincent Baeten ${ }^{\mathrm{c}}$, Juan Antonio Fernández \\ Pierna $^{\mathrm{c}}$ \\ ${ }^{a}$ Queens University Belfast, Institute for Global Food Security, Belfast, Northern Ireland, \\ $U K$ \\ ${ }^{b}$ Blendhub S.L., San Ginés, 30169 Murcia, Spain \\ ${ }^{c}$ Food and feed Quality Unit, Valorization of Agricultural Products Department, Walloon \\ Agricultural Research Centre (CRA-W), 24 Chaussée de Namur, 5030 Gembloux, Belgium \\ ${ }^{d}$ Queens University Belfast, Institute of Electronics, Communications and Information \\ Technology, Belfast, Northern Ireland, UK
}

\begin{abstract}
Hydrocolloids such as natural gums and carrageenans are used extensively in the food industry in various mixtures that are difficult to be characterised due to their similar chemical structure. The aim of this study was to develop an analytical framework for the identification and quantification of these compounds in complex mixtures using Near-infrared (NIR) spectroscopy and chemometrics. Partial Least Squares (PLS) regression accompanied by Continuous Locality Preserving Projections (CLPP) dimensionality reduction technique is proposed as chemometric framework. Four different analytical models based on this framework are developed and compared for the analysis of spectral fingerprints of food hydrocolloids mixtures. Classification results showed that this method allowed the discrimination of hydrocolloids in blends with a $100 \%$ of correct classification. The same scheme also allows the quantitative determination of the different types of food hydrocolloids (3 types) and/or their individual com-

\footnotetext{
${ }^{*}$ Corresponding author

Email addresses: kgeorgouli01@qub.ac.uk (Konstantia Georgouli), d.vincke@cra.wallonie.be (Damien Vincke), j.martinez-del-rincon@qub.ac.uk (Jesus Martinez Del Rincon), t.koidis@qub.ac.uk (Anastasios Koidis), v.baeten@cra.wallonie.be (Vincent Baeten), j.fernandez@cra.wallonie.be (Juan Antonio Fernández Pierna)
} 
pounds ( 8 different compounds) with a relative low root mean square error of prediction (RMSEP) of 0.028 and 0.038 respectively.

Keywords: Food hydrocolloids, Characterisation, NIR spectroscopy, CLPP, PLS

\section{Introduction}

2

Food hydrocolloids are hydrophilic polymers, mainly polysaccharides and proteins, which have the ability to change the food properties when dispersed in water [1, 2]. They are commonly used in the food industry as processing additives and more specifically as thickening (e.g. tara, guar, locust bean gum (LBG)) and gelling (e.g. carrageenan) agents due to their functional properties [3, 4. The individual hydrocolloids are usually prepared in blends for the creation of new food textures [1. The hydrocolloids are included in the legislation of the European Commission of approved food additives, and as a consequence, they need to be clearly labelled and distinguished when used in a product for quality control and/or labelling purposes [5]. With this aim in mind, a food company needs to certify that the pure products they received correspond to the specifications and there were not changes in the production line at the supplier factory from batch to batch. On the other hand, when producing blends at the very own company, they can have errors due to manipulation, labelling etc. so they need criteria to certify that the blend contains what is expected (from the company's specifications) and adhere to regulations. However, the different types of hydrocolloids share a common chemical structure which results in difficulties in characterising mixtures in the factory. This is coupled with the fact that they come usually in blends with or without excipients and added in small quantities in the final product formulation $(<2 \%)$ which all make their identification, and even more their quantification, very challenging [6]. Therefore, there is an increasing demand for rapid on-site characterisation of hydrocolloids as part of the quality control in the food industry.

The characterisation of the various hydrocolloids in a food industrial envi- 
ronment is mainly currently based on the measurement of different chemical parameters and physical parameters, such as viscosity, and the correlation of their values with the concentration of each compound [7. Different traditional physical, chemical and chromatographic analytical techniques have been used for the estimation of these parameters of hydrocolloids [3, 6, 8, [9]. Despite their accurate performance, these methods are time-consuming, destructive and demand expensive equipment and highly-skilled personnel which prevent its application to real-time and on-line screening of processes. Moreover, in these techniques, only specific compounds of interest are studied based on specific peaks in the instrumental responses (targeted analysis). In general, rapid, easy, non-destructive, multi-component and cost-effective analysis with minimal or none sample preparation can be rendered by infrared spectroscopy [10, 11]. However, the intrinsic nature of infrared spectroscopic techniques, combined with the fact that spectral overlapping usually occurs, can prevent the accurate quantification of different compounds [12, 13]. Thus, smart chemometric tools are demanded to enable the spectral characterisation of hydrocolloids in a hydrocolloid mixture.

Only a few papers have targeted the differentiation and quantification of gums [14, 15] and carrageenans [16, 7] using infrared spectroscopy in combination with chemometrics. Prado et al. 15] managed to differentiate carbohydrate gums, such as guar gum, locust bean gum and xanthan gum, and gum mixtures using diffuse reflectance (DRIFT) FT-MIR spectra coupled with Canonical variate analysis (CVA) with $100 \%$ accuracy. Moreover, they accomplished quantitative analysis of guar gum in locust bean gum using partial least squares (PLS) with a root mean square error of prediction (RMSEP) of 3.31. In another study, the discrimination of Acacia gum samples (Acacia senegal and Acacia seyal gums) was performed in the Principal Component Analysis (PCA) space after the application of extended multiplicative scatter correction (EMSC) pre-treatment to NIR spectra 14. Here again, PLS was used to establish a quantitative model for the analysis of Acacia senegal/Acacia seyal gum mixtures and the prediction of Acacia senegal in combination with interval PLS 
(iPLS) variable selection yielding a root mean square error of cross validation (RMSECV) of 0.053. Nevertheless, the mixtures investigated in the two aforementioned studies were only binary mixtures, i.e. with two types of gum. Regarding carrageenans, Prado-Fernández et al. [7] combined FTIR spectroscopy with PLS showing cross validation standard prediction errors (SEP) of 0.033, 0.033 and 0.042 for estimation of concentration levels of lambda, kappa and iota carrageenans respectively, in ternary standard carrageenan mixtures. RMSEP errors of about $0.04,0.03$ and 0.04 were obtained using a test set (half of the laboratory-prepared mixtures). Commercial carrageenans were characterised using single-reflection ATR FT-MIR spectrometer and principal component regression $(\mathrm{PCR})$ by predicting the total carrageenan content and the molar ratio of kappa and iota carrageenans with SEP of $5.6 \mathrm{~g} / 100 \mathrm{~g}$ and 6.1 and $6.6 \mathrm{~mol}$ $\%$, respectively [16]. As it can be noticed, in most of these works, PLS was applied [14, 15, 7]. However, no research endeavours have been reported for both detecting and quantifying simultaneously many compounds in a food hydrocolloid system such as gums, carrageenans and excipients due to the increasing complexity.

Recently, Continuous Locality Preserving Projections (CLPP) [17 has been introduced as a novel linear dimensionality reduction method that finds application in food analytical problems defined by continuous spectral data. This continuous modelling of the data allows CLPP to accurately represent mixtures and adulterations of food compounds by enhancing their interpretation, analysis, visualisation and classification. For this purpose, a systematic design of the training sample set (e.g. admixtures in a few concentration levels) is necessary to generate the desired continuity in the produced CLPP space.

The combination of CLPP with PLS has revealed an improvement in the performance of the simple PLS for the rapid detection of the adulteration of extra virgin olive oil with hazelnut oil [17]. However, no other analytical problem has been addressed with this innovative approach including the problem addressed in this paper.

Addressing a real need in the food production sector, the primary aim of 


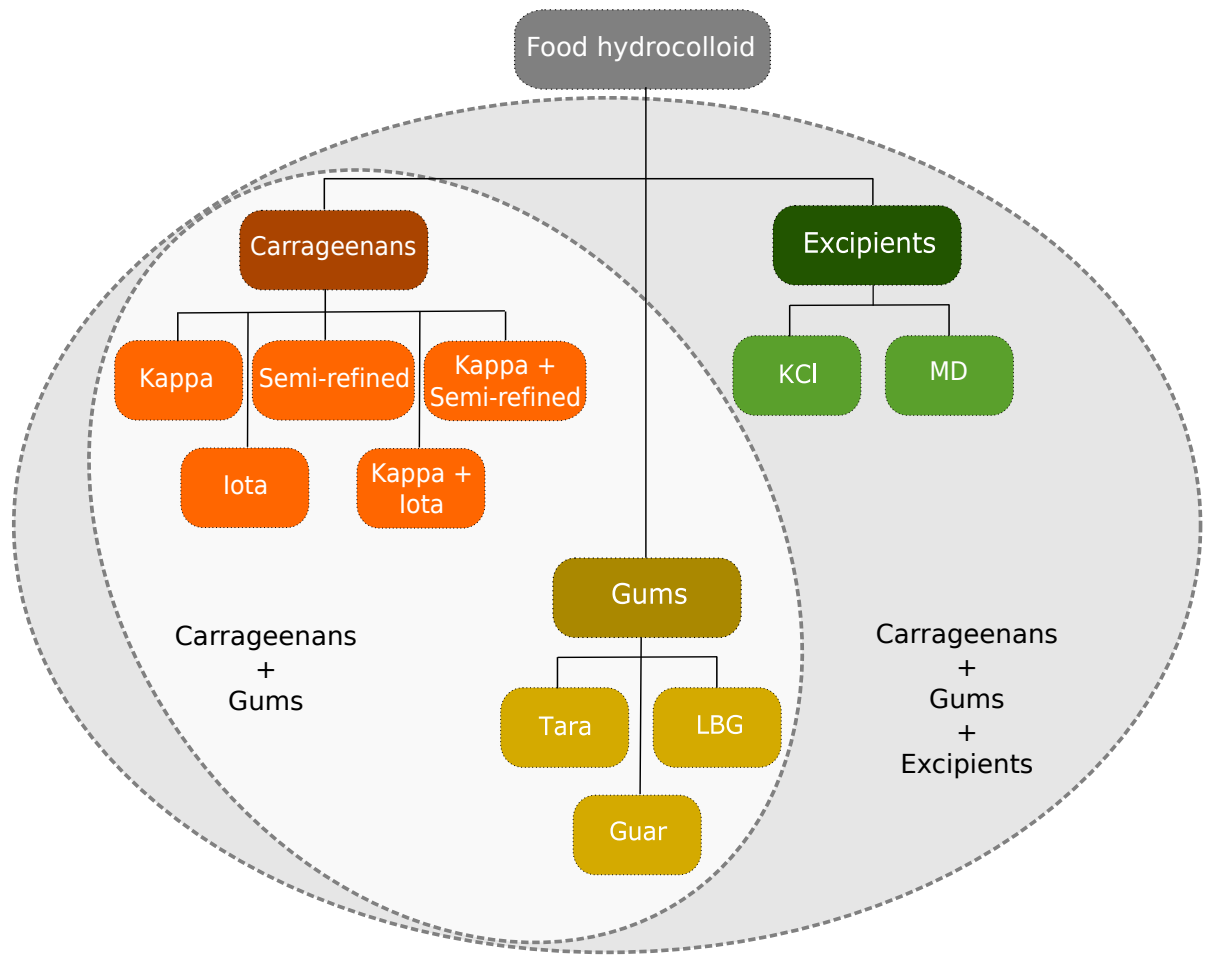

Fig. 1: Graphical representation of the food hydrocolloids in training dataset. LBG, Locust bean gum; KCl, Potassium chloride; MD: Maltodextrin. this work is to characterise complex food systems which contain blends of hydrocolloids (carageenans and gums) and excipients. To reach this aim, both

90 NIR spectroscopy and CLPP together with PLS are combined in our frame91 work. The main contribution of this paper is the use of CLPP in combination 92 with the hierarchical PLS models for the fine grain quantification, quantification 93 of subcategories belonging to the same basic category, in food mixtures that are 94 structurally complex. 


\section{Materials and methods}

\subsection{Samples}

Pure gums (tara, guar, locust bean gum (LBG)), pure excipients (Potassium chloride $(\mathrm{KCl})$, maltodextrin $(\mathrm{MD})$ ) and standardised carrageenans with $\mathrm{KCl}$ (kappa, iota, semi-refined) from different manufacturers were supplied by Blendhub. All these samples were characterised (purity, expiry date, etc.) and used for the preparation of carrageenan admixtures (kappa+iota and kappa+semirefined), carrageenans+gums and carrageenans+gums+excipients admixtures using several different batches, suppliers and certified blending protocols.

Specifically 29 samples (batch 1) were employed for the training of the chemometric models (see Table 11. Fig. 1 shows the structure of the available food hydrocolloids in the training dataset. The classification ability of the classification models was assessed by an additional dataset (batch 2) of 73 carraggeenan+gums+excipients admixtures (see Table A.1 in Appendix A). These samples were also especially prepared and supplied from Blendhub for this study and they as realistic representatives of the admixtures used in the factory for food production (real-world samples). These testing samples were only admixtures and included from four to eight individual compounds (Carrageenans: 46\%-69\%, gums: 20\%-35\% and excipients: 10\%-21.1\%, see Table A.1 in Appendix A. The admixture percentages were intentionally chosen to be different from training percentages to highlight the potential in realistic testing and diminish the effect of overfitting to the specific training combinations.

\subsection{Data acquisition}

Spectra were recorded using different NIR spectroscopic instruments. A NIR spectrometer model Foss XDS in the CRA-W laboratory was used for the spectral acquisition of training samples. The NIR spectra were recorded in the wavelength region of $400-2498 \mathrm{~nm}$ with a resolution of $2 \mathrm{~nm}$ resulting in 1050 variables. For each sample two ring cups have been filled with the sample and measured in order to obtain two replicates per sample. Both replicates 
Table 1: Composition of the food hydrocolloid samples that have been used in training stage of the study (batch1).

\begin{tabular}{|c|c|c|c|c|c|c|c|c|}
\hline \multirow[b]{2}{*}{ Sample } & \multicolumn{3}{|c|}{ CARRAGEENANS (\%) } & \multicolumn{3}{|c|}{ GUMS (\%) } & \multicolumn{2}{|c|}{ EXCIPIENTS (\%) } \\
\hline & Kappa & Iota & $\begin{array}{l}\text { Semi- } \\
\text { refined }\end{array}$ & Tara & Guar & LBG & $\mathrm{KCl}$ & MD \\
\hline 1 & 100 & 0 & 0 & 0 & 0 & 0 & 0 & 0 \\
\hline 2 & 0 & 100 & 0 & 0 & 0 & 0 & 0 & 0 \\
\hline 3 & 0 & 0 & 100 & 0 & 0 & 0 & 0 & 0 \\
\hline 4 & 0 & 0 & 0 & 100 & 0 & 0 & 0 & 0 \\
\hline 5 & 0 & 0 & 0 & 0 & 100 & 0 & 0 & 0 \\
\hline 6 & 0 & 0 & 0 & 0 & 0 & 100 & 0 & 0 \\
\hline 7 & 0 & 0 & 0 & 0 & 0 & 0 & 100 & 0 \\
\hline 8 & 0 & 0 & 0 & 0 & 0 & 0 & 0 & 100 \\
\hline 9 & 80 & 20 & 0 & 0 & 0 & 0 & 0 & 0 \\
\hline 10 & 60 & 0 & 40 & 0 & 0 & 0 & 0 & 0 \\
\hline 11 & 85 & 0 & 0 & 5 & 5 & 5 & 0 & 0 \\
\hline 12 & 0 & 85 & 0 & 5 & 5 & 5 & 0 & 0 \\
\hline 13 & 80 & 0 & 0 & 20 & 0 & 0 & 0 & 0 \\
\hline 14 & 80 & 0 & 0 & 0 & 20 & 0 & 0 & 0 \\
\hline 15 & 80 & 0 & 0 & 0 & 0 & 20 & 0 & 0 \\
\hline 16 & 70 & 0 & 0 & 20 & 0 & 10 & 0 & 0 \\
\hline 17 & 70 & 0 & 0 & 0 & 20 & 10 & 0 & 0 \\
\hline 18 & 60 & 20 & 0 & 20 & 0 & 0 & 0 & 0 \\
\hline 19 & 0 & 20 & 60 & 0 & 20 & 0 & 0 & 0 \\
\hline 20 & 60 & 20 & 0 & 0 & 0 & 20 & 0 & 0 \\
\hline 21 & 55 & 0 & 0 & 5 & 5 & 5 & 30 & 0 \\
\hline 22 & 55 & 0 & 0 & 5 & 5 & 5 & 0 & 30 \\
\hline 23 & 0 & 55 & 0 & 5 & 5 & 5 & 30 & 0 \\
\hline 24 & 0 & 55 & 0 & 5 & 5 & 5 & 0 & 30 \\
\hline 25 & 0 & 55 & 0 & 0 & 15 & 0 & 0 & 30 \\
\hline 26 & 0 & 55 & 0 & 0 & 15 & 0 & 30 & 0 \\
\hline 27 & 55 & 0 & 0 & 0 & 0 & 20 & 25 & 0 \\
\hline 28 & 0 & 55 & 0 & 0 & 0 & 20 & 25 & 0 \\
\hline 29 & 0 & 0 & 55 & 0 & 0 & 20 & 25 & 0 \\
\hline
\end{tabular}

LBG: Locust bean gum; KCl: Potassium chloride; MD: Maltodextrin. 
were used for the chemometric model construction. The testing samples were acquired $ө$-site between 400nm and $2499.5 \mathrm{~nm}$ using a Foss DS2500 instrument the same NIR spectrometer which has with a spectral resolution of 0.5 $\mathrm{nm}$ (4200 variables). The different spectral resolution was selected in order to avoid, again, any overfitting of our model to the acquisition tool.

\subsection{Data pre-treatment}

The visible and shortwave near-infrared region (400-1098 nm) was cut out from the original spectra of 1050 variables because of the very little useful information in this region. The resulting NIR spectra (700 variables) were preprocessed using Standard Normal Variate (SNV) [18, followed by detrend [18] and S-Golay filter [19, 20] [polynomial order $=2$, frame size=9], which were applied for removing the scatter, correcting the baseline and smoothing the data points, respectively (see Fig. 2). As can be seen in Fig. 2 which shows the same spectra, before and after preprocessing, some spectra such of the excipients are flipped after the pre-treatment where a clear discrimination of the different types of excipients versus the gums and carrageenans is revealed. This can be verified visually by observing the Fig. 2 that shows the same spectra, before and after preprocessing, with different colours depending on the compound that samples belong. Moreover, a differentiation of the carrageenan+gum+excipient samples between carrageenan + gum $+\mathrm{KCl}$ and carrageenan + gum $+\mathrm{MD}$ samples is also noticeable. Regarding the testing phase, before the same data pre-treatment, linear interpolation was applied to the test spectra in order to get the desirable number of variables $(n=1050)$ since they were acquired with a spectrometer with different spectral resolution [21, 22] to reduce overfitting to the NIR calibration set.

All chemometric preprocessing was performed by means of in-house Matlab routines (The MathWorks Inc., USA).

\subsection{Continuous Locality Preserving Projections (CLPP)}

CLPP 17] is a semi-supervised linear method for dimensionality reduction which learns manifolds designated for continuous data. It is an extension of the 


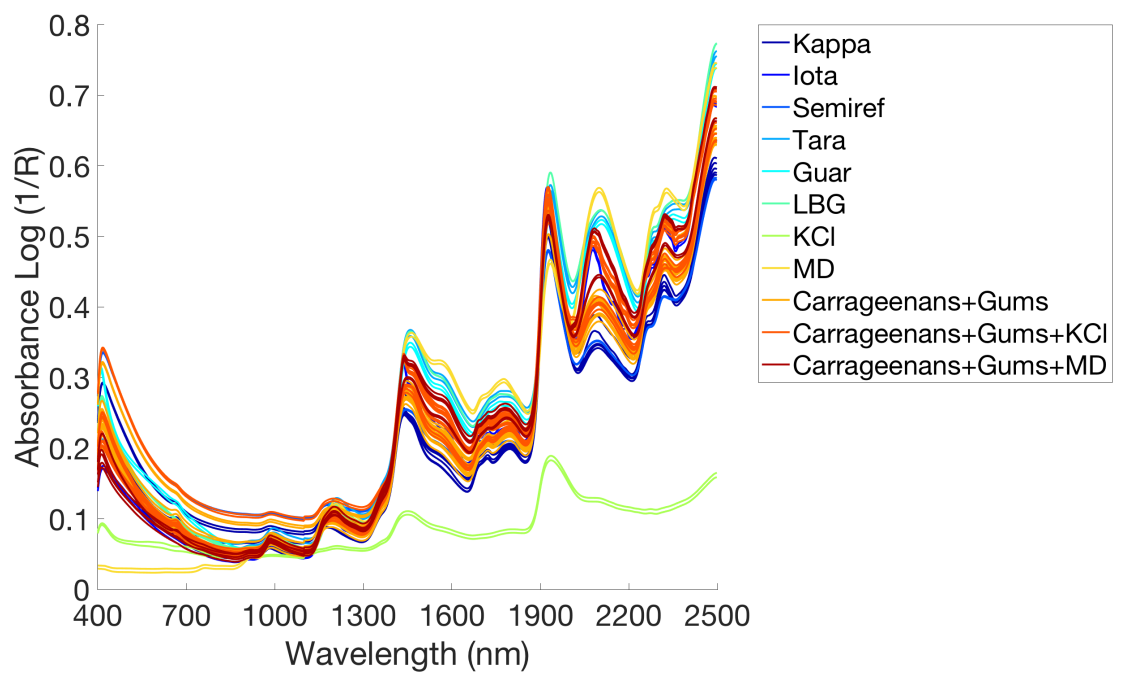

(a)

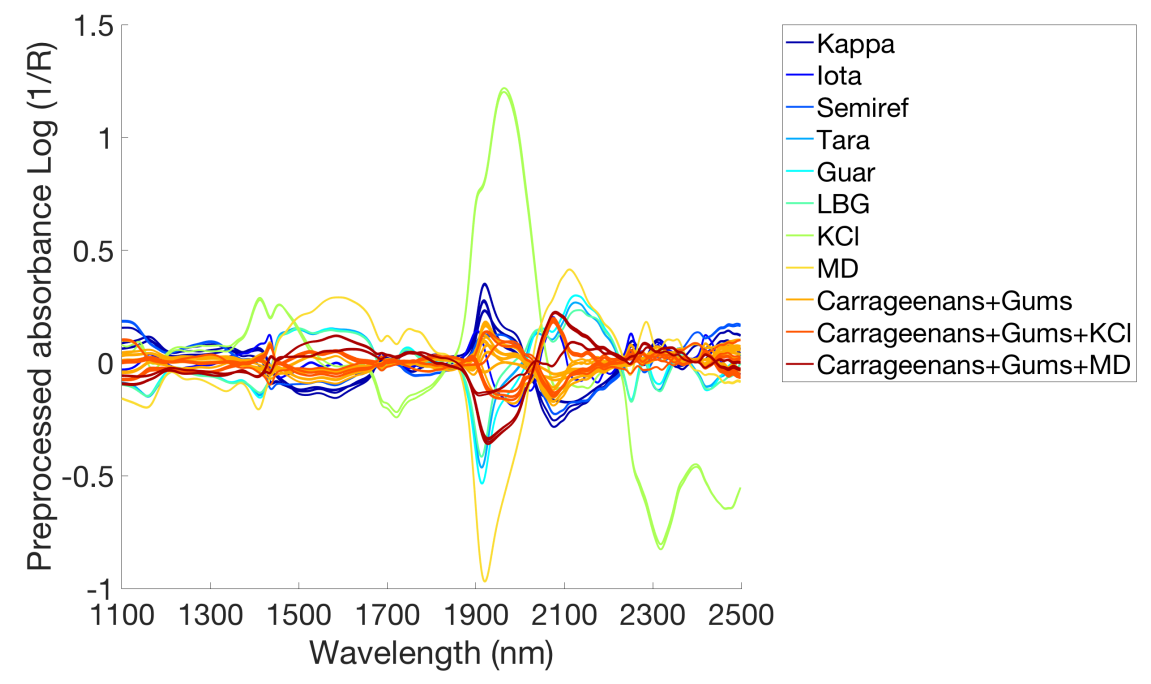

(b)

Fig. 2: NIR spectra of pure carrageenans (Kappa, Iota and Semiref), gums (Tara, Guar and $\mathrm{LBG}$ ), excipients ( $\mathrm{KCl}$ and $\mathrm{MD}$ ), carrageenans and gums admixtures, carrageenans, gums and $\mathrm{KCl}$ admixtures and carrageenans, gums and MD admixtures: (a) Raw data; (b) Preprocessed data. LBG: Locust bean gum; KCl: Potassium chloride; MD: Maltodextrin. 


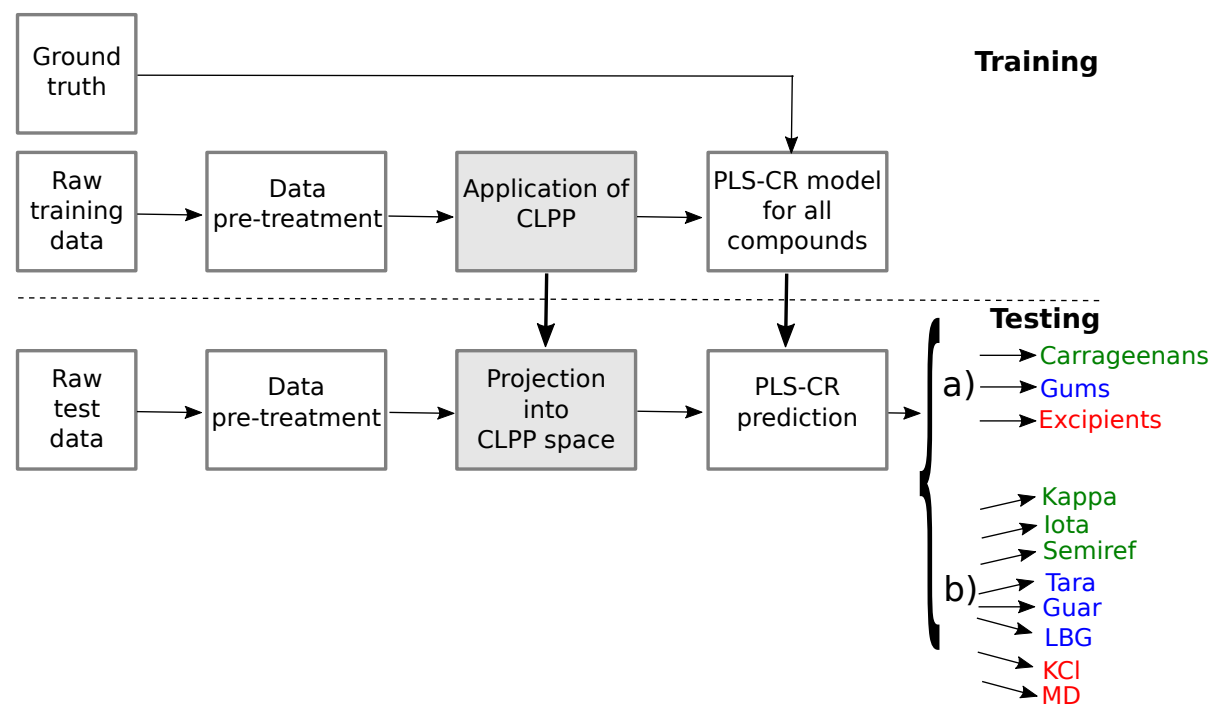

Fig. 3: Experimental design of CLPP+PLS-CR (a) with 3 values for the prediction of totals and (b) with 8 values for the prediction of all individual compounds.

linear dimensionality reduction technique Locality Preserving Projections (LPP) 23. preserving continuity as in previous non-linear techniques such as Temporal Laplacian Eigenmaps (TLE) 24. Given a set of $Y=y_{1}, y_{2}, \cdots, y_{n}$ data points in high dimensional space $\left(y_{k} \in R^{D}\right)$, CLPP is able to transform this into its low dimensional space by mapping it to a set of points $Z=m_{1}, m_{2}, \cdots, m_{n}$ ( $m_{k} \in R^{d}$ ) with $d \ll D$, while preserving the continuity of the data. CLPP is based on the construction of two different neighbourhood graphs to preserve data continuity which express implicitly continuity dependencies between data points. Specifically, two continuous neighbourhoods are produced for each data point:

(a) Continuous neighbourhood $(C)$ : the previous and next closest points in sequence of current data point.

(a) Similarity neighbourhood $(S)$ : the points parallel to current data point in the parallel trajectories. Each trajectory is generated by the same number of continuous neighbours. 


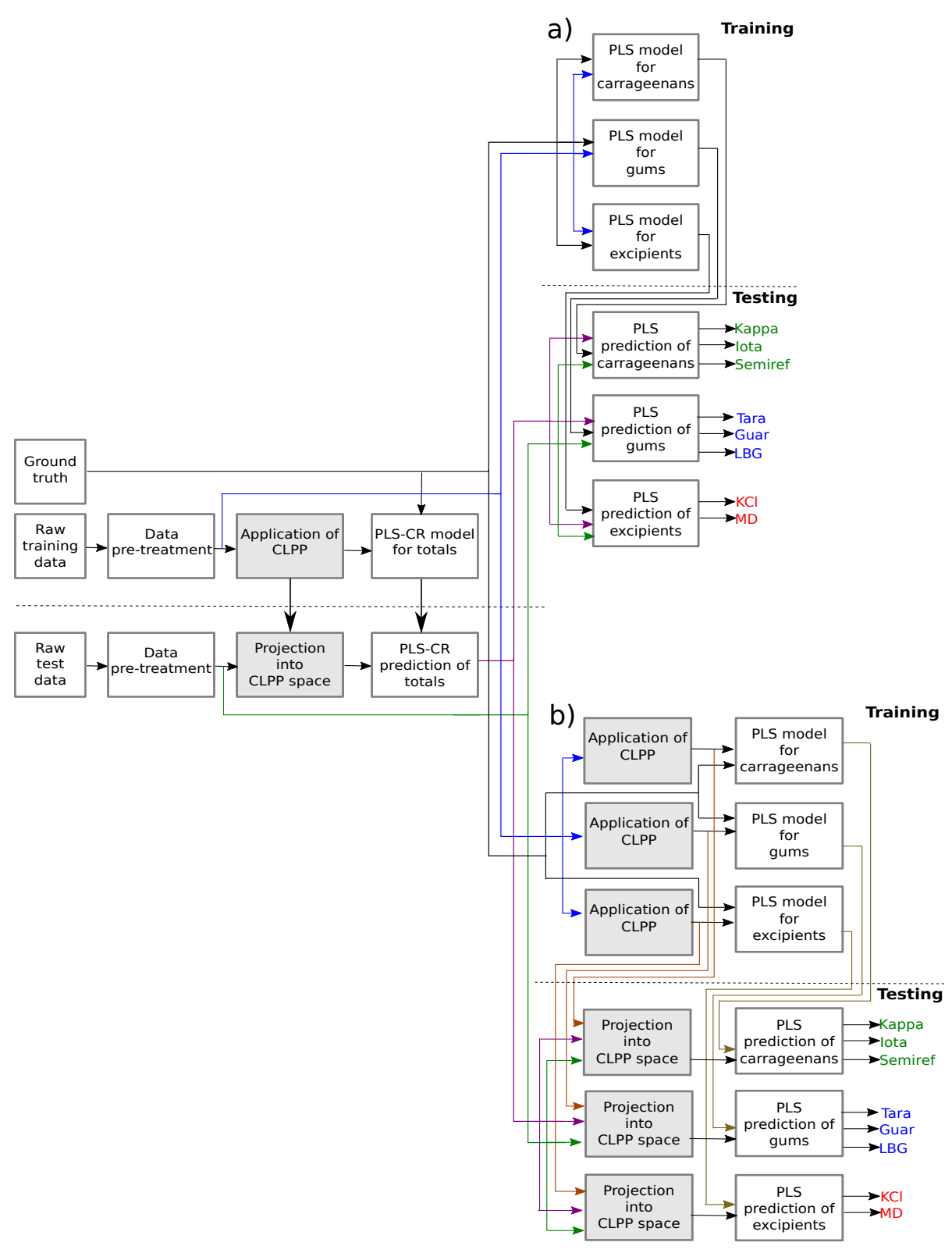

Fig. 4: Experimental design of double stage approaches for the prediction of all individual compounds (a) an initial CLPP+PLS-CR model discriminating the 3 main groups and classifying the mixtures, followed by 3 specific PLS models; (b) an initial CLPP+PLS-CR model discriminating the 3 main groups and classifying the mixtures, followed by 3 specific CLPP+PLS models. 
The process of dimensionality reduction can be summarised briefly by the following steps. First, weights $\mathrm{W}$ are assigned to the edges of each graph $\mathrm{G} \in\{C, S\}$ using the standard LPP formulation. Then the eigenvectors $V$ of embedded space are computed. The $d$ eigenvectors $V^{*}$ with the smallest nonzero eigenvalues make the embedded space. These eigenvectors and eigenvalues are calculated by solving the generalized eigenvalue problem:

$$
\arg \min _{V^{*}}\left(V^{T} \cdot Y^{T} \cdot\left(L_{C}+\beta \cdot L_{S}\right) \cdot Y \cdot V\right)
$$

subject to

$$
V^{T} \cdot Y^{T} \cdot\left(D_{C}+\beta \cdot D_{S}\right) \cdot Y \cdot V=1
$$

where $L_{C}=D_{C}-G_{C}$ and $L_{S}=D_{S}-G_{S}$ are the Laplacian matrices and $D_{C}$ and $D_{S}$ are diagonal matrices. $\beta$ is a weighting factor for balancing the continuous and similarity variabilities. Regarding other continuous techniques, CLPP has the advantages of being simple and providing automatically both directional mapping (from low to high and from high to low dimensional spaces) while reducing the space.

\section{Experimental setup}

Based on the sample composition of food hydrocolloids, the training data are divided into five classes for identification purposes, 1: pure carrageenan, 2: pure gum, 3: pure excipient, 4: carrageenan+gum admixture and 5: carrageenan+gum+excipient admixture (Fig. 1). The great difficulty of this problem hinders an experimental design with higher class resolution. Initially, the performance of the Partial least squares discriminant analysis (PLS-DA), kNearest Neighbors (kNN) and support vector machines (SVM) have been calculated to this 5-class problem as the most used classification methods. These supervised classification techniques were applied to the original (pre-processed) data for qualitative analysis only.

However, to meet the main goal of this study regarding the characterisation of complex hydrocolloid mixtures, quantitative models between hydrocolloid 
composition and NIR spectral data were established using PLS. Here, apart from the conventional usage of PLS as a regression technique, PLS is also expanded for the characterisation of the mixtures. The main feature of PLS-CR is that it calculates the predicted concentrations using PLS regression and assigns a class membership to each testing sample during prediction. Specifically, if the PLS output value of a testing sample for a specific compound is positive then this compound is present to this specific sample. Then, based on the present compounds in a testing sample, the sample is classified to one of the five classes by applying classification rules after PLS. A classification rule is a condition related to the present compounds in a testing sample which allows the assignment of this sample to a model class when this condition is met. The PLS-CR input variables are a set with the number of dimensions resulting from a dimensionality reduction technique prior PLS-CR, such as CLPP or PCA, and the number of latent variables used for the development of the PLS model (see Table A.2 in Appendix A. In case of no dimensionality reduction technique applied before PLS-CR, all variables of raw spectra are used. From now on in this article, we call this variant of PLS as Partial Least Squares regression with Classification Rules (or PLS-CR).

The characterisation of food hydrocolloid blends is investigated using two different scenarios with respect to the expected model behaviour. In the first and more straightforward scenario, only the total concentration of carrageenans, gums and excipients are predicted (3 response values for a testing sample) using a CLPP+PLS-CR model (see Fig. 3a). In the second scenario, the individual compounds of carrageenans (kappa, iota, semi-refined), gums (tara, guar, locust bean gum), and excipients (Potassium chloride, maltodextrin) are predicted. In this challenging scenario, three approaches are tested: a) a CLPP+PLS-CR model able to provide predicted values for all 8 compounds (see Fig. 3b)), b) a double stage approach, where an initial CLPP+PLS-CR model discriminates between the 3 main groups and classifies the mixtures, which are further quantified by 3 specific PLS models (See Fig. 4 a), and c) a double stage approach, where an initial CLPP+PLS-CR model for the discrimination between the 3 
main groups is followed by 3 specific CLPP + PLS models for the quantification of all the individual compounds (See Fig. $4 \mathrm{p}$ ).

After data pre-treatment, CLPP has been applied to all the aforementioned cases for reducing the high dimensional space of the data while modeling the continuity of the data. All the aforementioned cases are mainly differentiated by the different PLS/PLS-CR models generated after CLPP. According to CLPP theory [17, each hydrocolloid mixture will be modelled as a data series, where each data point $m_{k}$ is the low dimensional representation of each high dimensional spectrum at different concentration grades of two compounds from $0 \%$ to $100 \%$. Since the mixtures can include until 8 compounds, the concentration grades of two of the compounds are changing while the rest of them are kept the same. Adjacent data points $m_{k-t}$ and $m_{k+t}$, composing the continuous neighbourhood $\left(C_{k}\right)$, will be the same mixture but at the immediate higher and lower concentration levels for the one component correspondingly and inversely for the other. The similarity neighbourhood $\left(S_{k}\right)$ will be the set of different hydrocolloid admixtures at the exact same number of concentration grades. As mentioned previously, about PLS/PLS-CR, positive response for a total/compound means that the specific hydrocolloid blend contains the specific type/compound. Thereafter, the normalisation of PLS/PLS-CR responses was carried out in order that their sum equals 1.

The performance of the proposed schemes is evaluated by comparing them with the simple PLS and PCR. It is also compared against other pattern recognition techniques that they have been proposed in the literature [14, 15] and we consider they have potential to tackle this characterisation problem. These were PCA+PLS, and Canonical variate analysis (CVA)+PLS and Multivariate Curve Resolution-Alternating Least Squares (MCR-ALS) 25. Moreover, it should be noted that the same rationale behind discriminant PLS with classification rules is applied also to PCR (PCR with classification rules or PCR-CR) and MCR-ALS (MCR-ALS with classification rules or MCR-ALS-CR). MCRALS calculations were performed using MCR-ALS GUI 2.0 26] and setting correlation constraints. All MCR-ALS models were performed with correlation 
constraint for the three totals simultaneously, for the eight compounds simultaneously and for the components of each group simultaneously (double stage) and non-negativity constraint in concentrations. The estimation of MCR-ALS components was done by using singular value decomposition (SVD). The initial estimates were obtained by means of the estimation of purest variables. Moreover, in the case of MCR-ALS, regarding pre-treatment, only the visible and shortwave near-infrared region was cut out because because the other pre-treatment steps did not allow to achieve convergence during the iterative optimisation. Correct classification rate and RMSEP were used as model performance parameters to evaluate the results. RMSEP values were calculated for each individual compound in isolation and for the overall admixture estimation. Tuning parameters have been empirically set for the building of the chemometric models (see Table A.2 in Appendix A.

\section{Results and discussion}

\subsection{Classification and regression results}

Thanks to the ability of the CLPP to model the continuous nature of the admixtures as data series, models can be created with only a few (but representative) samples (here 29 samples). Fig. 5 shows the capability of CLPP to discriminate not only the groups but even the individual compounds in the same space.

Widely applied and leading multivariate classification techniques such as PLS-DA and kNN exhibited the weakest classification performance in this 5class problem (Table 2). This proves the difficulty and complexity of this problem. SVM is the only classification technique used for qualitative analysis which achieved a 100\% classification rate due to its ability to model data non-linearity present in these complex mixtures. Despite the fact SVM demonstrated a good performance and can be used for quantitative analysis, its results lack transparency since there is no resulting vectors like scores and loadings for visualisation purposes and the tuning of its parameters is a complex task 27. For these 


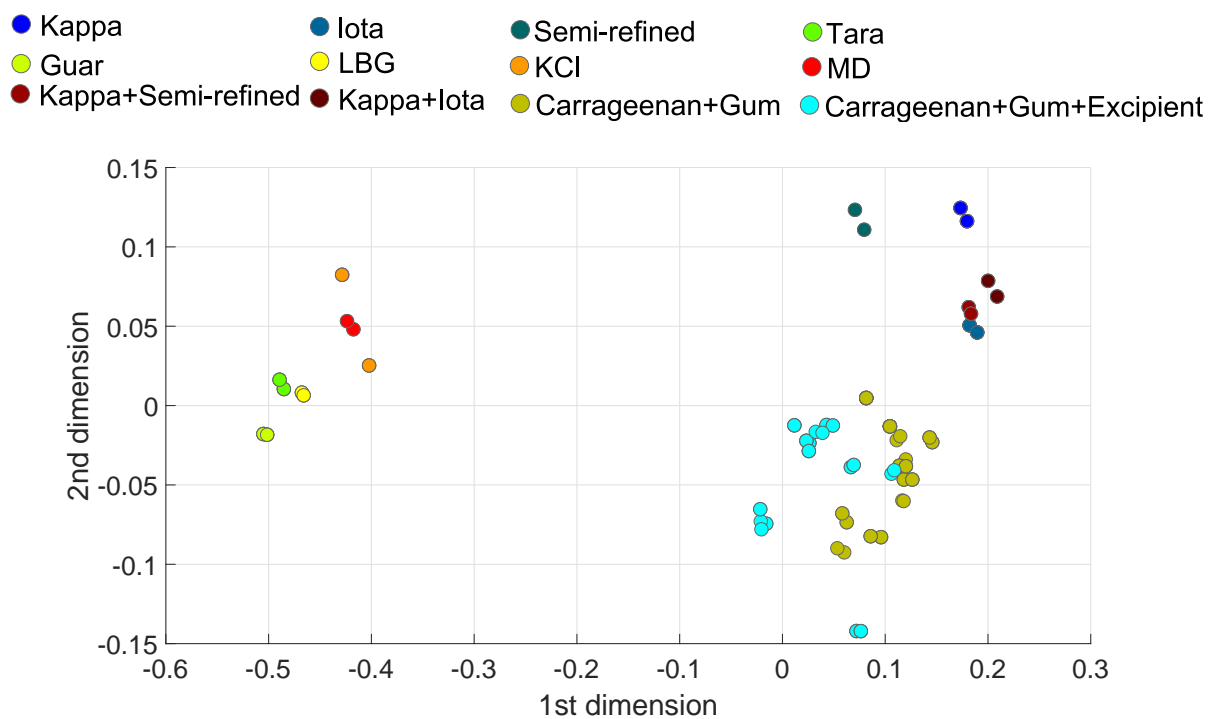

Fig. 5: CLPP space of NIR data by colouring all the individual compounds. LBG: Locust bean gum; $\mathrm{KCl}$ : Potassium chloride; MD: Maltodextrin.

reasons, PLS was selected for the creation of quantitative models for the remaining experiments since it is the most used method for building a calibration model for quantitative analysis (commercial software and in-house routines). Classification results using quantitative models showed that apart from MCRALS-CR, all of the investigated pattern recognition techniques based on NIR data allowed the discrimination between food hydrocolloid blends with a $100 \%$ of correct classification for all the cases (Table 3). The less accurate classification performance of MCR-ALS-CR is due to the intrinsic difficulties of MCR-ALS to resolve and quantify the compounds contributing very little to the measured spectra 28, 29. Specifically, MCR-ALS-CR failed to properly quantify the excipients, which have the lowest concentration levels in hydrocolloid mixtures, and therefore some carrageenan+gum+excipient admixtures were wrongly classified as carrageenan+gum admixtures.

However, regression results are better evaluation metrics for this analytical problem where high precision regarding the composition of hydrocolloid compounds is required by the industry. Here, CLPP+PLS-CR outperforms all the 
Table 2: Classification rate (\%) (TP/Total testing samples) for the classification of hydrocolloid mixtures for the 5 -class problem using NIR spectral data.

\begin{tabular}{ll}
\hline $\begin{array}{l}\text { CLASSIFICATION } \\
\text { TECHNIQUE }\end{array}$ & Classification rate $(\%)$ (TP/Total testing samples) \\
\hline PLS-DA & $73.97(54 / 73)$ \\
\hline $\mathrm{kNN}$ & $83.56(61 / 73)$ \\
\hline $\mathrm{SVM}$ & $100.00(73 / 73)$ \\
\hline
\end{tabular}

TP: True positives; PLS-DA: Partial least squares discriminant analysis; kNN: k-Nearest Neighbors; SVM: Support vector machines.

other pattern recognition techniques in all cases. Specifically, CLPP+PLS-CR allows the quantitative determination of the different kinds of food hydrocolloids (totals) with a RMSEP of 0.028 (Table 4). Interestingly, our scheme achieves half the RMSEP of the next better performance, the ones of CVA+PLS-CR.

Regarding the prediction and quantification of all the individual compounds (8 different compounds) in a food hydrocolloid mixture, the use of a PLSCR/PCR-CR model with 8 output values gives higher RMSEP values caused by the higher number of dependent variables that PLS-CR/PCR-CR has to predict (Table 4). Again here, PLS-CR+CLPP was generally better giving the lower RMSEP. Interestingly, the double stage approaches, where the initial problem is split hierarchically into smaller and simpler problems (an initial PLS-CR/PCR-CR model quantifying the three groups of compounds followed by three PLS/PCR models, one per group of compounds), achieved a better overall RMSEP than the PLS-CR/PCR-CR models with 8 output values in all the cases (Table 5). CLPP+PLS-CR followed by three PLS models (1st double stage approach) showed a better performance in the quantification of all compounds, RMSEP $=0.045$ in testing dataset, compared with the other techniques. This performance was improved even more by applying CLPP before the three PLS models (2nd double stage approach) for quantifying the indi- 
Table 3: Classification rate (\%) (TP/Total testing samples) for the prediction of totals and of individual hydrocolloid compounds classification of hydrocolloid mixtures for a 5-class problem using NIR spectral data by quantifying the totals and/or the individual hydrocolloid compounds.

\begin{tabular}{lccc}
\hline CLASSIFICATION & Classification rate $(\%)$ (TP/Total testing samples) \\
TECHNIQUE & & Prediction of all individual compounds \\
\cline { 3 - 4 } & Prediction of & PLS (8 output & $\begin{array}{c}\text { Double stage } \\
\text { totals }\end{array}$ \\
& $100.00(73 / 73)$ & $100.00(73 / 73)$ & $100.00(73 / 73)$ \\
\hline PLS-CR & $100.00(73 / 73)$ & $100.00(73 / 73)$ & $100.00(73 / 73)$ \\
\hline PCR-CR & $87.67(64 / 73)$ & $91.78(67 / 73)$ & $87.67(64 / 73)$ \\
\hline MCR-ALS-CR & $100.00(73 / 73)$ & $100.00(73 / 73)$ & $100.00(73 / 73)$ \\
\hline PCA+PLS-CR & $100.00(73 / 73)$ & $100.00(73 / 73)$ & $100.00(73 / 73)$ \\
\hline CVA+PLS-CR & $100.00(73 / 73)$ & $100.00(73 / 73)$ & $100.00(73 / 73)$ \\
\hline CLPP+PLS-CR & & approaches $)$ \\
\hline
\end{tabular}

TP: True positives; PLS: Partial least squares regression; PLS-CR: Partial least squares regression with classification rules; PCR-CR: Principal component regression with classification rules; MCR-ALS-CR: Multivariate Curve Resolution-Alternating Least Squares with classification rules; PCA: Principal Component Analysis; CVA: Canonical variate analysis; CLPP: Continuous locality preserving projections.

vidual compounds (Overall RMSEP $=0.038$ ) which proves that dimensionality reduction using CLPP helps the fine grain quantification. However, this further improvement does not happen for the other two dimensionality techniques (PCA and CVA) where RMSEP was either slightly improved or worsen. Table6 shows some examples of the quantitative values predicted by the best approach model (2nd double stage approach using CLPP+PLS-CR/CLPP+PLS). As can be seen, the predicted values are pretty close to the real ones with a small error. 
Table 4: Root mean squared error for the prediction of totals and individual compounds using NIR spectral data by applying PLS-CR/PCR-CR with 3 and 8 output values, respectively.

\begin{tabular}{|c|c|c|c|c|c|c|}
\hline \multirow[t]{2}{*}{ RMSEP } & \multicolumn{6}{|c|}{ Classification technique } \\
\hline & PLS-CR & PCR-CR & $\begin{array}{c}\text { MCR- } \\
\text { ALS-CR }\end{array}$ & $\begin{array}{l}\text { PCA + } \\
\text { PLS-CR }\end{array}$ & $\begin{array}{l}\text { CVA + } \\
\text { PLS-CR }\end{array}$ & $\begin{array}{l}\text { CLPP + } \\
\text { PLS-CR }\end{array}$ \\
\hline \multicolumn{7}{|c|}{ Prediction of totals ( 3 output values) } \\
\hline RMSEP_Carrageenan & 0.062 & 0.066 & 0.071 & 0.062 & 0.036 & 0.030 \\
\hline RMSEP_Gum & 0.076 & 0.073 & 0.081 & 0.075 & 0.055 & 0.025 \\
\hline RMSEP_Excipient & 0.037 & 0.038 & 0.126 & 0.037 & 0.071 & 0.030 \\
\hline Overall RMSEP & 0.061 & 0.061 & 0.096 & 0.061 & 0.056 & 0.028 \\
\hline \multicolumn{7}{|c|}{ Prediction of all individual compounds ( 8 output values) } \\
\hline RMSEP_Kappa & 0.067 & 0.047 & 0.146 & 0.047 & 0.057 & 0.055 \\
\hline RMSEP_Iota & 0.070 & 0.063 & 0.038 & 0.063 & 0.067 & 0.042 \\
\hline RMSEP_Semi-refined & 0.108 & 0.091 & 0.162 & 0.091 & 0.141 & 0.068 \\
\hline RMSEP_Tara & 0.069 & 0.058 & 0.115 & 0.058 & 0.076 & 0.090 \\
\hline RMSEP_Guar & 0.078 & 0.078 & 0.131 & 0.078 & 0.082 & 0.092 \\
\hline RMSEP_LBG & 0.052 & 0.062 & 0.141 & 0.062 & 0.077 & 0.066 \\
\hline RMSEP_KCl & 0.135 & 0.124 & 0.100 & 0.124 & 0.128 & 0.071 \\
\hline RMSEP_MD & 0.043 & 0.018 & 0.089 & 0.018 & 0.040 & 0.066 \\
\hline Overall RMSEP & 0.083 & 0.074 & 0.121 & 0.074 & 0.090 & 0.071 \\
\hline
\end{tabular}

PLS-CR: Partial least squares regression with classification rules; PCR-CR: Principal component regression with classification rules; MCR-ALS-CR: Multivariate Curve Resolution-Alternating Least Squares with classification rules; PCA: Principal Component Analysis; CVA: Canonical variate analysis; CLPP: Continuous locality preserving projections; LBG: Locust bean gum; KCl: Potassium chloride; MD: Maltodextrin. 
Table 5: Root mean squared error for the prediction of individual compounds using NIR spectral data by applying the two approaches of double stage.

\begin{tabular}{|c|c|c|c|c|c|c|}
\hline \multirow{2}{*}{$\begin{array}{l}\text { RMSEP } \\
\text { First approach }\end{array}$} & \multicolumn{5}{|c|}{$\begin{array}{l}\text { Classification technique for the totals / } \\
\text { Regression technique for all the compounds }\end{array}$} & \multirow[b]{2}{*}{$\begin{array}{c}\text { CLPP + } \\
\text { PLS-CR } \\
\text { / PLS }\end{array}$} \\
\hline & $\begin{array}{l}\text { PLS-CR } \\
\text { / PLS }\end{array}$ & $\begin{array}{l}\text { PCR-CR } \\
\text { / PCR }\end{array}$ & $\begin{array}{l}\text { MCR- } \\
\text { ALS-CR }\end{array}$ & $\begin{array}{c}\text { PCA + } \\
\text { PLS-CR } \\
/ \text { PLS }\end{array}$ & $\begin{array}{c}\text { CVA + } \\
\text { PLS-CR } \\
\text { / PLS }\end{array}$ & \\
\hline RMSEP_Kappa & 0.038 & 0.038 & 0.405 & 0.037 & 0.035 & 0.032 \\
\hline RMSEP_Iota & 0.057 & 0.069 & 0.270 & 0.057 & 0.045 & 0.048 \\
\hline RMSEP_Semi-refined & 0.044 & 0.041 & 0.267 & 0.044 & 0.040 & 0.041 \\
\hline RMSEP_Tara & 0.058 & 0.045 & 0.248 & 0.058 & 0.059 & 0.044 \\
\hline RMSEP_Guar & 0.078 & 0.073 & 0.140 & 0.078 & 0.054 & 0.061 \\
\hline RMSEP_LBG & 0.050 & 0.068 & 0.147 & 0.050 & 0.056 & 0.046 \\
\hline RMSEP_KCl & 0.045 & 0.043 & 0.082 & 0.045 & 0.064 & 0.046 \\
\hline RMSEP_MD & 0.039 & 0.038 & 0.082 & 0.039 & 0.040 & 0.037 \\
\hline Overall RMSEP & 0.053 & 0.054 & 0.230 & 0.052 & 0.050 & 0.045 \\
\hline Second approach & $\begin{array}{l}\text { PLS-CR } \\
\text { / PLS }\end{array}$ & $\begin{array}{l}\text { PCR-CR } \\
\text { / PCR }\end{array}$ & $\begin{array}{l}\text { MCR- } \\
\text { ALS-CR }\end{array}$ & $\begin{array}{c}\text { PCA + } \\
\text { PLS-CR } \\
/ \text { PCA } \\
+ \text { PLS }\end{array}$ & $\begin{array}{c}\text { CVA + } \\
\text { PLS-CR } \\
\text { / CVA } \\
+ \text { PLS }\end{array}$ & $\begin{array}{c}\text { CLPP + } \\
\text { PLS-CR } \\
/ \text { CLPP } \\
+ \text { PLS }\end{array}$ \\
\hline RMSEP_Kappa & $\mathrm{n} / \mathrm{a}$ & $\mathrm{n} / \mathrm{a}$ & $\mathrm{n} / \mathrm{a}$ & 0.033 & 0.034 & 0.034 \\
\hline RMSEP_Iota & $\mathrm{n} / \mathrm{a}$ & $\mathrm{n} / \mathrm{a}$ & $\mathrm{n} / \mathrm{a}$ & 0.056 & 0.035 & 0.038 \\
\hline RMSEP_Semi-refined & $\mathrm{n} / \mathrm{a}$ & $\mathrm{n} / \mathrm{a}$ & $\mathrm{n} / \mathrm{a}$ & 0.043 & 0.055 & 0.032 \\
\hline RMSEP_Tara & $\mathrm{n} / \mathrm{a}$ & $\mathrm{n} / \mathrm{a}$ & $\mathrm{n} / \mathrm{a}$ & 0.061 & 0.060 & 0.041 \\
\hline RMSEP_Guar & $\mathrm{n} / \mathrm{a}$ & $\mathrm{n} / \mathrm{a}$ & $\mathrm{n} / \mathrm{a}$ & 0.070 & 0.055 & 0.043 \\
\hline RMSEP_LBG & $\mathrm{n} / \mathrm{a}$ & $\mathrm{n} / \mathrm{a}$ & $\mathrm{n} / \mathrm{a}$ & 0.053 & 0.052 & 0.043 \\
\hline RMSEP_KCl & $\mathrm{n} / \mathrm{a}$ & $\mathrm{n} / \mathrm{a}$ & $\mathrm{n} / \mathrm{a}$ & 0.042 & 0.059 & 0.042 \\
\hline RMSEP_MD & $\mathrm{n} / \mathrm{a}$ & $\mathrm{n} / \mathrm{a}$ & $\mathrm{n} / \mathrm{a}$ & 0.038 & 0.047 & 0.031 \\
\hline Overall RMSEP & $\mathrm{n} / \mathrm{a}$ & $\mathrm{n} / \mathrm{a}$ & $\mathrm{n} / \mathrm{a}$ & 0.051 & 0.051 & 0.038 \\
\hline
\end{tabular}

PLS: Partial least squares regression; PLS-CR: Partial least squares regression with classification rules; PCR: Principal component regression; $26^{R}-\mathrm{CR}$ : Principal component regression with classification rules; MCR-ALS-CR: Multivariate Curve Resolution-Alternating Least Squares with classification rules; PCA: Principal Component Analysis; CVA: Canonical variate analysis; CLPP: Continuous locality preserving projections; LBG: Locust bean gum; KCl: Potassium chloride; MD: Maltodextrin; n/a: not applicable. 
Table 6: Predictions of a few testing samples made by the best approach model using CLPP+PLS-CR/CLPP+PLS (double stage).

\begin{tabular}{|c|c|c|c|c|c|c|c|c|}
\hline \multirow[b]{2}{*}{$\begin{array}{l}\text { Testing } \\
\text { sample }\end{array}$} & \multicolumn{3}{|c|}{ CARRAGEENANS (\%) } & \multicolumn{3}{|c|}{ GUMS (\%) } & \multicolumn{2}{|c|}{ EXCIPIENTS $(\%)$} \\
\hline & Kappa & Iota & $\begin{array}{l}\text { Semi- } \\
\text { refined }\end{array}$ & Tara & Guar & LBG & $\mathrm{KCl}$ & MD \\
\hline $\begin{array}{l}\text { Actual } \\
(\# 20)\end{array}$ & 49.5 & 0 & 17.5 & 16.5 & 0 & 6.2 & 8.2 & 2.1 \\
\hline $\begin{array}{l}\text { Predicted } \\
(\# 20)\end{array}$ & 49.3 & 0 & 17.2 & 11.8 & 0 & 10.6 & 8.1 & 3 \\
\hline $\begin{array}{l}\text { Error } \\
(\# 20)\end{array}$ & 0.2 & $\mathbf{0}$ & 0.3 & 4.7 & 0 & 4.4 & 0.1 & 0.9 \\
\hline $\begin{array}{l}\text { Actual } \\
(\# 33)\end{array}$ & 42 & 6 & 0 & 4 & 26 & 2 & 6 & 14 \\
\hline $\begin{array}{l}\text { Predicted } \\
(\# 33)\end{array}$ & 36 & 13.2 & 0 & 4 & 26.2 & 1.3 & 3.3 & 16 \\
\hline $\begin{array}{l}\text { Error } \\
(\# 33)\end{array}$ & 6 & 7.2 & 0 & 0 & 0.2 & 0.7 & 2.7 & 2 \\
\hline $\begin{array}{l}\text { Actual } \\
(\# 43)\end{array}$ & 3 & 30 & 18 & 21 & 0 & 8 & 10 & 10 \\
\hline $\begin{array}{l}\text { Predicted } \\
(\# 43)\end{array}$ & 10.1 & 26.6 & 15 & 17.5 & 0 & 12.2 & 8 & 10.6 \\
\hline $\begin{array}{l}\text { Error } \\
(\# 43)\end{array}$ & 7.1 & 3.4 & 3 & 3.5 & 0 & 4.2 & 2 & 0.6 \\
\hline $\begin{array}{l}\text { Actual } \\
(\# 66)\end{array}$ & 54.5 & 0 & 2 & 8.1 & 8.1 & 8.1 & 2 & 17.2 \\
\hline $\begin{array}{l}\text { Predicted } \\
(\# 66)\end{array}$ & 54.7 & 0 & 2.6 & 7.5 & 8.4 & 11.4 & 1.8 & 13.6 \\
\hline $\begin{array}{l}\text { Error } \\
(\# 66)\end{array}$ & 0.2 & 0 & 0.6 & 0.6 & 0.3 & 3.3 & 0.2 & 3.6 \\
\hline $\begin{array}{l}\text { Actual } \\
(\# 67)\end{array}$ & 47 & 5 & 0 & 16 & 16 & 0 & 8 & 8 \\
\hline $\begin{array}{l}\text { Predicted } \\
(\# 67)\end{array}$ & 47.4 & 6 & 0 & 15.1 & 14.3 & 0 & 6.1 & 11.1 \\
\hline $\begin{array}{l}\text { Error } \\
(\# 67)\end{array}$ & 0.4 & 1 & 0 & 0.9 & 1.7 & 0 & 1.9 & 3.1 \\
\hline
\end{tabular}

LBG: Locust bean gum; KCl: Potassium chloride; MD: Maltodextrin. 
Remarkably, this approach does not only identify correctly all the individual compounds in the admixtures, it also quantifies them. Its downside, however, is that the selection of the proper values for the tuning of CLPP parameters is a complex and time-consuming process since more than one parameter have to be optimised. Nevertheless, procedures for the automatic optimisation of the tuning parameters is something we intend to study in our future work. In order to obtain a robust model and diminish the risk of overfitting, the admixture percentages in testing dataset were intentionally selected to be different from training percentages and different spectral resolution was used for the training and testing dataset. About NIR based calibration models, future work should include more variation by using hydrocolloids from different geographic origins. Overall To sum up, the application of CLPP as a dimensionality reduction technique prior the PLS-CR has showed up to around $54 \%$ improvement in RMSEP of the simple PLS-CR for the prediction of the totals and decreased up to $28 \%$ the RMSEP of PLS-CR/PLS using the second double stage approach, which confirms the great potentiality of CLPP+PLS-CR and CLPP+PLS in combination with the double stage approach for the statistical analysis of NIR spectroscopic data in the characterisation of a hydrocolloid system and in tackling compositional analysis problems in general. While our results are promising and show good generalisation to new mixture percentages and spectral resolutions, the small amount of samples used to train the models, especially given their much larger dimensionality, may results in overfitted models. In future work, we aim to extract a larger order of magnitude in the number of samples to improve the learned models.

\section{Conclusions}

In this study, an analytical framework of CLPP coupled with PLS was developed for addressing the characterisation of complex food hydrocolloid admixtures using NIR spectroscopic data. Results showed that although the relatively small number of training samples and, at the same time, the high number of 


\section{Acknowledgements}

This research was supported with funding from The Department Learning and Employment Northern Ireland (DELNI) (PhD studentship block grant) and through a Training \& Mobility Funding Programme from Safefood (Ireland). Special thanks to Stéphane Brichard and Sandrine Mauro who collected the Foss XDS data for CRA-W. 


\section{Appendix A}

Table A.1: Composition of food hydrocolloids in testing dataset.

\begin{tabular}{|c|c|c|c|c|c|c|c|c|}
\hline & \multicolumn{3}{|c|}{ CARRAGEENANS (\%) } & \multicolumn{3}{|c|}{ GUMS (\%) } & \multicolumn{2}{|c|}{ EXCIPIENTS (\%) } \\
\hline Sample & Kappa & Iota & $\begin{array}{l}\text { Semi- } \\
\text { refined }\end{array}$ & Tara & Guar & LBG & $\mathrm{KCl}$ & MD \\
\hline 1 & 55 & 0 & 0 & 10 & 10 & 10 & 15 & 0 \\
\hline 2 & 0 & 55 & 0 & 0 & 30 & 0 & 7.5 & 7.5 \\
\hline 3 & 0 & 0 & 55 & 6 & 0 & 24 & 0 & 15 \\
\hline 4 & 30 & 0 & 25 & 30 & 0 & 0 & 5 & 10 \\
\hline 5 & 55 & 0 & 0 & 0 & 0 & 30 & 12 & 3 \\
\hline 6 & 0 & 30 & 25 & 6 & 20 & 4 & 3 & 12 \\
\hline 7 & 8 & 0 & 50 & 7 & 0 & 20 & 15 & 0 \\
\hline 8 & 48 & 0 & 0 & 32 & 0 & 0 & 10 & 10 \\
\hline 9 & 0 & 50 & 6 & 9 & 9 & 10 & 0 & 16 \\
\hline 10 & 0 & 63 & 0 & 0 & 0 & 25 & 6 & 6 \\
\hline 11 & 40 & 11 & 0 & 10 & 0 & 21 & 14 & 4 \\
\hline 12 & 30 & 0 & 17 & 0 & 35 & 0 & 4 & 14 \\
\hline 13 & 31.4 & 0 & 31.4 & 22.5 & 0 & 4 & 7.8 & 2.9 \\
\hline 14 & 22 & 22 & 22 & 20 & 1 & 2 & 6 & 5 \\
\hline 15 & 7 & 0 & 50 & 0 & 24 & 0 & 10 & 9 \\
\hline 16 & 4 & 16 & 38 & 2 & 2 & 24 & 14 & 0 \\
\hline 17 & 10 & 0 & 40 & 23 & 9 & 0 & 9 & 9 \\
\hline 18 & 30 & 28 & 0 & 5 & 20 & 0 & 11 & 6 \\
\hline 19 & 22 & 42 & 0 & 1 & 25 & 0 & 5 & 5 \\
\hline 20 & 49.5 & 0 & 17.5 & 16.5 & 0 & 6.2 & 8.2 & 2.1 \\
\hline 21 & 20 & 16 & 15 & 8 & 0 & 25 & 0 & 16 \\
\hline 22 & 30 & 3 & 20 & 2 & 9 & 18 & 18 & 0 \\
\hline 23 & 0 & 20.8 & 38.6 & 0 & 0 & 20.8 & 9.9 & 9.9 \\
\hline 24 & 30 & 28 & 0 & 20 & 11 & 0 & 11 & 0 \\
\hline 25 & 8 & 18 & 36 & 0 & 25 & 2 & 0 & 11 \\
\hline 26 & 20.8 & 27.7 & 0 & 19.8 & 14.9 & 0 & 0 & 16.8 \\
\hline 27 & 17 & 0 & 38 & 15 & 10 & 5 & 10 & 5 \\
\hline 28 & 16 & 14 & 30 & 0 & 0 & 29 & 0 & 11 \\
\hline 29 & 0 & 0 & 52 & 3 & 25 & 0 & 12 & 8 \\
\hline 30 & 40 & 11 & 0 & 0 & 2 & 29 & 6 & 12 \\
\hline 31 & 0 & 36 & 26 & 22 & 3 & 0 & 7 & 6 \\
\hline 32 & 28 & 32 & 0 & 28 & 0 & 0 & 8 & 4 \\
\hline 33 & 42 & 6 & 0 & 4 & 26 & 2 & 6 & 14 \\
\hline 34 & 48 & 0 & 15 & 10 & 14 & 0 & 3 & 10 \\
\hline 35 & 38 & 0 & 17 & 0 & 2 & 33 & 5 & 5 \\
\hline 36 & 24 & 0 & 40 & 6 & 5 & 11 & 10 & 4 \\
\hline 37 & 16 & 24 & 11 & 0 & 22 & 11 & 5 & 11 \\
\hline 38 & 0 & 49 & 14 & 0 & 18 & 9 & 5 & 5 \\
\hline 39 & 47.5 & 8.9 & 0 & 5 & 6.9 & 13.9 & 17.8 & 0 \\
\hline 40 & 49 & 0 & 9 & 16 & 2 & 5 & 0 & 19 \\
\hline 41 & 69 & 0 & 0 & 6 & 6 & 9 & 0 & 10 \\
\hline 42 & 40 & 10 & 0 & 5 & 22 & 5 & 15 & 3 \\
\hline 43 & 3 & 30 & 18 & 21 & 0 & 8 & 10 & 10 \\
\hline 44 & 9 & 0 & 48 & 8 & 0 & 20 & 5 & 10 \\
\hline 45 & 0 & 45 & 2 & 0 & 32 & 2 & 11 & 8 \\
\hline
\end{tabular}


Table A.1 (Continued)

\begin{tabular}{|l|l|l|l|l|l|l|l|l|}
\hline & \multicolumn{2}{|l}{ CARRAGEENANS (\%) } & \multicolumn{3}{|c|}{ GUMS (\%) } & \multicolumn{2}{l|}{ EXCIPIENTS (\%) } \\
\hline Sample & Kappa & Iota & $\begin{array}{l}\text { Semi- } \\
\text { refined }\end{array}$ & Tara & Guar & LBG & KCI & MD \\
\hline 46 & 0 & 3 & 50 & 0 & 4 & 28 & 15 & 0 \\
\hline 47 & 20 & 20 & 20 & 24 & 0 & 0 & 2 & 14 \\
\hline 48 & 0 & 15.6 & 33.3 & 0 & 10 & 20 & 13.3 & 7.8 \\
\hline 49 & 60 & 5 & 0 & 18 & 2 & 0 & 5 & 10 \\
\hline 50 & 5 & 60 & 0 & 4 & 20 & 0 & 6 & 5 \\
\hline 51 & 3 & 0 & 54 & 3 & 0 & 28 & 12 & 0 \\
\hline 52 & 0 & 0 & 67 & 0 & 0 & 22 & 11 & 0 \\
\hline 53 & 52 & 0 & 0 & 29 & 0 & 0 & 19 & 0 \\
\hline 54 & 0 & 64.6 & 0 & 0 & 20.2 & 0 & 0 & 15.2 \\
\hline 55 & 5 & 50 & 0 & 2 & 26 & 0 & 0 & 17 \\
\hline 56 & 0 & 6 & 45 & 0 & 6 & 28 & 3 & 12 \\
\hline 57 & 21 & 21 & 21 & 0 & 12 & 12 & 13 & 0 \\
\hline 58 & 4 & 44 & 8 & 14 & 14 & 0 & 0 & 16 \\
\hline 59 & 40 & 8 & 4 & 16 & 0 & 16 & 8 & 8 \\
\hline 60 & 0 & 0 & 46 & 0 & 0 & 35 & 19 & 0 \\
\hline 61 & 60 & 2 & 0 & 12 & 12 & 2 & 12 & 0 \\
\hline 62 & 5 & 55 & 0 & 5 & 17 & 0 & 12 & 6 \\
\hline 63 & 0 & 5 & 44 & 0 & 5 & 30 & 12 & 4 \\
\hline 64 & 17 & 17 & 17 & 17 & 17 & 0 & 12 & 3 \\
\hline 65 & 0 & 57.8 & 6.9 & 0 & 17.6 & 6.9 & 0 & 10.8 \\
\hline 66 & 54.5 & 0 & 2 & 8.1 & 8.1 & 8.1 & 2 & 17.2 \\
\hline 67 & 47 & 5 & 0 & 16 & 16 & 0 & 8 & 8 \\
\hline 68 & 0 & 65 & 0 & 0 & 22 & 0 & 13 & 0 \\
\hline 69 & 45 & 10 & 0 & 22 & 5 & 0 & 9 & 9 \\
\hline 70 & 20 & 20 & 16 & 11 & 0 & 22 & 0 & 11 \\
\hline 71 & 25 & 0 & 25 & 0 & 31 & 0 & 16 & 3 \\
\hline 72 & 0 & 0 & 65 & 0 & 11 & 11 & 13 & 0 \\
\hline 73 & 5 & 56 & 0 & 3 & 0 & 22 & 12 & 2 \\
\hline & & & & & & & & \\
\hline & & & & & & \\
\hline & & & & & & & \\
\hline
\end{tabular}

LBG: Locust bean gum; $\mathrm{KCl}$ : Potassium chloride; MD: Maltodextrin. 
Table A.2: Parameters values used in measurements for gathering results for the characterisation of a food hydrocolloid system.

\begin{tabular}{|c|c|c|c|c|}
\hline $\begin{array}{c}\text { Classification } \\
\text { Regression } \\
\text { technique }\end{array}$ & \multicolumn{4}{|c|}{ Parameters values } \\
\hline \multicolumn{5}{|c|}{ For qualitative analysis } \\
\hline PLS-DA & \multicolumn{4}{|l|}{$\mathrm{Lv}=5$} \\
\hline $\mathrm{kNN}$ & \multicolumn{4}{|l|}{$\mathrm{K}=3$} \\
\hline SVM & \multicolumn{4}{|l|}{$\operatorname{sigma}=6, C=1$} \\
\hline \multicolumn{5}{|c|}{ For qualitative and quantitative analysis } \\
\hline & \multirow{2}{*}{$\begin{array}{c}\text { Prediction } \\
\text { of totals }\end{array}$} & \multicolumn{3}{|c|}{ Prediction of all individual compounds } \\
\hline & & 1st approach & 2nd approach & 3rd approach \\
\hline PLS-CR & $\mathrm{Lv}=2$ & $\mathrm{Lv}=14$ & $\begin{array}{c}\text { Totals: } \mathrm{Lv}=2, \\
\text { carrageenan: } \\
\mathrm{Lv}=15 \text {, gums: } \\
\mathrm{Lv}=12 \text {, excipients: } \\
\mathrm{Lv}=10\end{array}$ & $\mathrm{n} / \mathrm{a}$ \\
\hline PCR-CR & pcr_comps $=2$ & pcr_comps $=17$ & $\begin{array}{c}\text { Totals: } \\
\text { pcr_comps=2, } \\
\text { carrageenan: } \\
\text { pcr_comps }=18, \\
\text { gums: } \\
\text { pcr_comps }=10, \\
\text { excipients: } \\
\text { pcr_comps }=12\end{array}$ & $\mathrm{n} / \mathrm{a}$ \\
\hline MCR-ALS-CR & $\begin{array}{c}\text { mcr- } \\
\text { als_comps }=6\end{array}$ & $\begin{array}{c}\text { mcr- } \\
\text { als_comps }=8\end{array}$ & $\begin{array}{c}\text { Totals: } \\
\text { mcr-als_comps }=6, \\
\text { carrageenan: } \\
\text { mcr-als_comps }=5, \\
\text { gums: } \\
\text { mcr-als_comps }=5, \\
\text { excipients: } \\
\text { mcr-als_comps }=5\end{array}$ & $\mathrm{n} / \mathrm{a}$ \\
\hline
\end{tabular}


Table A.2 (Continued)

\begin{tabular}{|c|c|c|c|c|}
\hline \multirow{2}{*}{$\begin{array}{c}\text { Classification } \\
\text { Regression } \\
\text { technique }\end{array}$} & \multirow{2}{*}{$\begin{array}{c}\text { Prediction } \\
\text { of totals }\end{array}$} & \multicolumn{3}{|c|}{ Prediction of all individual compounds } \\
\hline & & 1st approach & 2nd approach & 3rd approach \\
\hline $\mathrm{PCA}+\mathrm{PLS}-\mathrm{CR}$ & $\begin{array}{c}\text { Pca_dims }=3 \\
L v=2\end{array}$ & $\begin{array}{c}\text { Pca_dims }=17, \\
L v=17\end{array}$ & $\begin{array}{c}\text { Totals: } \\
\text { Pca_dims }=3, \mathrm{Lv}=2, \\
\text { carrageenan: } \\
\mathrm{Lv}=15 \text {, gums: } \\
\mathrm{Lv}=12 \text {, excipients: } \\
\mathrm{Lv}=10\end{array}$ & $\begin{array}{c}\text { Totals: } \\
\text { Pca_dims }=3, \mathrm{Lv}=2 \\
\text { carrageenan: } \\
\text { Pca_dims }=25, \\
\text { Lv }=15, \text { gums: } \\
\text { Pca_dims }=15, \\
\text { Lv=12, excipients: } \\
\text { Pca_dims }=12, \\
\text { Lv }=11\end{array}$ \\
\hline CVA + PLS-CR & $\begin{array}{c}\text { Pca_dims }=13, \\
\text { cva_dims }=6, \\
L v=2\end{array}$ & $\begin{array}{c}\text { Pca_dims }=12, \\
\text { cva_dims }=10, \\
L v=10\end{array}$ & $\begin{array}{c}\text { Totals: } \\
\text { Pca_dims }=13, \\
\text { cva_dims }=6, \mathrm{Lv}=2 \\
\text { carrageenan: } \\
\text { Lv=15, gums: } \\
\text { Lv=12, excipients: } \\
\text { Lv }=8\end{array}$ & $\begin{array}{c}\text { Totals: } \\
\text { Pca_dims }=13, \\
\text { cva_dims }=6, \mathrm{Lv}=2 \\
\text { carrageenan: } \\
\text { Pca_dims }=20, \\
\text { cva_dims }=16, \\
\text { Lv=16, gums: } \\
\text { Pca_dims }=13, \\
\text { cva_dims }=12, \\
\text { Lv=11, excipients: } \\
\text { Pca_dims }=21, \\
\text { cva_dims }=16, \text { Lv }=6\end{array}$ \\
\hline
\end{tabular}


Table A.2 (Continued)

\begin{tabular}{|c|c|c|c|c|}
\hline \multirow{2}{*}{$\begin{array}{c}\text { Classification } \\
\text { Regression } \\
\text { technique }\end{array}$} & \multirow{2}{*}{$\begin{array}{c}\text { Prediction } \\
\text { of totals }\end{array}$} & \multicolumn{3}{|c|}{ Prediction of all individual compounds } \\
\hline & & 1st approach & 2nd approach & 3rd approach \\
\hline $\begin{array}{l}\text { CLPP+ } \\
\text { PLS-CR }\end{array}$ & $\begin{array}{c}\text { clpp_dims }=2, \\
\text { pca_ratio }=0.85, \\
\mathrm{t}=3, \mathrm{r}=4, \\
\beta=1.15, \\
\text { smoothsize }=4, \\
\mathrm{Lv}=2\end{array}$ & $\begin{array}{c}\text { clpp_dims }=8, \\
\text { pca_ratio }=0.972, \\
\mathrm{t}=4, \mathrm{r}=3, \\
\beta=0.62, \\
\text { smoothsize }=1, \\
\mathrm{Lv}=5\end{array}$ & $\begin{array}{c}\text { Totals: } \\
\text { clpp_dims }=2, \\
\text { pca_ratio }=0.85, \\
\mathrm{t}=3, \mathrm{r}=4, \beta=1.15, \\
\text { smoothsize }=4, \\
\mathrm{Lv}=2, \\
\text { carrageenan: } \\
\mathrm{Lv}=15 \text {, gums: } \\
\mathrm{Lv}=12, \text { excipients: } \\
\mathrm{Lv}=10\end{array}$ & $\begin{array}{c}\text { Totals: } \\
\text { clpp_dims }=2, \\
\text { pca_ratio }=0.85, \\
\text { t=3, r= }=4, \beta=1.15, \\
\text { smoothsize }=4, \\
\text { Lv }=2 \\
\text { carrageenan: } \\
\text { clpp_dims }=18, \\
\text { pca_ratio }=0.995, \\
\text { t=2, r=9, } \beta=1.13, \\
\text { smoothsize }=8, \\
\text { Lv }=11, \text { gums: } \\
\text { clpp_dims }=11, \\
\text { pca_ratio }=0.992, \\
\text { t=4, r=13, } \beta=0.68, \\
\text { smoothsize }=4, \\
\text { Lv=9 }, \text { excipients: } \\
\text { clpp_dims }=12, \\
\text { pca_ratio }=0.987, \\
\text { t=5, r }=11, \beta=0.24, \\
\text { smoothsize }=6, \\
\text { Lv }=7\end{array}$ \\
\hline
\end{tabular}

Lv: latent variables; K: Number of nearest neighbors; sigma: the sigma factor in the Gaussian radial basis function kernel; C: box constraint, the Lagrange multipliers are bounded to be within the range $[0, \mathrm{C}]$; n/a: not applicable; pcr_comps: the number of PCR components; mcr-als_comps: the number of MCR-ALS components; clpp_dims: the dimensionality of the reduced CLPP subspace; pca_ratio: the percentage of principal component kept in the PCA step. The percentage is calculated based on the eigenvalue. Default is 1 where all the non-zero eigenvalues will be kept; $t$ : the number of nearest points in sequence; $\mathrm{r}$ : the number of parallel points; $\beta$ : weighting factor for balancing the continuous and similarity variabilities; smoothsize: smoothing factor which is used for the creation of the similarity neighbourhood. The bigger this value is, the smoother the points will be. 


\section{References}

[1] D. Saha, S. Bhattacharya, Hydrocolloids as thickening and gelling agents in food: a critical review, Journal of food science and technology 47 (6) (2010) 587-597.

[2] P. Burey, B. Bhandari, T. Howes, M. Gidley, Hydrocolloid gel particles: formation, characterization, and application, Critical reviews in food science and nutrition 48 (5) (2008) 361-377.

[3] C. Cevoli, F. Balestra, L. Ragni, A. Fabbri, Rheological characterisation of selected food hydrocolloids by traditional and simplified techniques, Food hydrocolloids 33 (1) (2013) 142-150.

[4] S. Barak, D. Mudgil, Locust bean gum: processing, properties and food applicationsa review, International journal of biological macromolecules 66 (2014) 74-80.

[5] Off. J. Eur. Union L295/1, Regulation (EC) No 1333/2008 of the European Parliament and of the Council by establishing a Union list of food additives, http://eur-lex.europa.eu/legal-content/EN/ TXT/?qid=1490368600372\&uri=CELEX :32011R1129, [Online; accessed 24March-2017] (2011).

[6] M. A. Roberts, B. Quemener, Measurement of carrageenans in food: challenges, progress, and trends in analysis, Trends in food science \& technology 10 (4) (1999) 169-181.

[7] J. Prado-Fernández, J. Rodrıguez-Vázquez, E. Tojo, J. Andrade, Quantitation of $\kappa$-, $\iota$-and $\lambda$-carrageenans by mid-infrared spectroscopy and pls regression, Analytica Chimica Acta 480 (1) (2003) 23-37. 
[8] V. L. Campo, D. F. Kawano, D. B. da Silva, I. Carvalho, Carrageenans: Biological properties, chemical modifications and structural analysis-a review, Carbohydrate Polymers 77 (2) (2009) 167-180.

[9] I. Gashua, P. Williams, M. Yadav, T. Baldwin, Characterisation and molecular association of nigerian and sudanese acacia gum exudates, Food Hydrocolloids 51 (2015) 405-413.

[10] J. Moros, S. Garrigues, M. de la Guardia, Vibrational spectroscopy provides a green tool for multi-component analysis, TrAC Trends in Analytical Chemistry 29 (7) (2010) 578-591.

[11] A. Ropodi, E. Panagou, G.-J. Nychas, Data mining derived from food analyses using non-invasive/non-destructive analytical techniques; determination of food authenticity, quality \& safety in tandem with computer science disciplines, Trends in Food Science \& Technology 50 (2016) 11-25.

[12] K. Javidnia, M. Parish, S. Karimi, B. Hemmateenejad, Discrimination of edible oils and fats by combination of multivariate pattern recognition and ft-ir spectroscopy: a comparative study between different modeling methods, Spectrochimica Acta Part A: Molecular and Biomolecular Spectroscopy 104 (2013) 175-181.

[13] E. Sánchez-López, M. Sánchez-Rodríguez, A. Marinas, J. Marinas, F. Urbano, J. Caridad, M. Moalem, Chemometric study of andalusian extra virgin olive oils raman spectra: Qualitative and quantitative information, Talanta 156 (2016) 180-190.

[14] Y. Dong, K. M. Sørensen, S. He, S. B. Engelsen, Gum arabic authentication and mixture quantification by near infrared spectroscopy, Food Control 78 (2017) 144-149.

[15] B. M. Prado, S. Kim, B. F. Özen, L. J. Mauer, Differentiation of carbohydrate gums and mixtures using fourier transform infrared spectroscopy 
and chemometrics, Journal of agricultural and food chemistry 53 (8) (2005) $2823-2829$.

[16] P. Volery, R. Besson, C. Schaffer-Lequart, Characterization of commercial carrageenans by fourier transform infrared spectroscopy using singlereflection attenuated total reflection, Journal of agricultural and food chemistry 52 (25) (2004) 7457-7463.

[17] K. Georgouli, J. M. Del Rincon, A. Koidis, Continuous statistical modelling for rapid detection of adulteration of extra virgin olive oil using mid infrared and raman spectroscopic data, Food chemistry 217 (2017) 735-742.

[18] R. J. Barnes, M. S. Dhanoa, S. J. Lister, Standard normal variate transformation and de-trending of near-infrared diffuse reflectance spectra, Appl. Spectrosc. 43 (5) (1989) 772-777.

URL http://as .osa.org/abstract.cfm?URI=as-43-5-772

[19] A. Savitzky, M. J. Golay, Smoothing and differentiation of data by simplified least squares procedures., Analytical chemistry 36 (8) (1964) 16271639.

[20] J. Steinier, Y. Termonia, J. Deltour, Smoothing and differentiation of data by simplified least square procedure, Analytical Chemistry 44 (11) (1972) 1906-1909.

[21] K. Georgouli, M. T. Osorio, J. Martinez Del Rincon, A. Koidis, Data augmentation in food science: Synthesising spectroscopic data of vegetable oils for performance enhancement, Journal of Chemometrics 32 (6) (2018) e3004.

[22] K. Diaz-Chito, K. Georgouli, A. Koidis, J. M. del Rincon, Incremental model learning for spectroscopy-based food analysis, Chemometrics and Intelligent Laboratory Systems 167 (2017) 123-131.

[23] X. He, P. Niyogi, Locality preserving projections, in: Advances in neural information processing systems, 2004, pp. 153-160. 
[24] M. Lewandowski, J. Martinez-del Rincon, D. Makris, J.-C. Nebel, Temporal extension of laplacian eigenmaps for unsupervised dimensionality reduction of time series, in: 2010 20th International Conference on Pattern Recognition, IEEE, 2010, pp. 161-164.

[25] R. Tauler, Multivariate curve resolution applied to second order data, Chemometrics and intelligent laboratory systems 30 (1) (1995) 133-146.

[26] J. Jaumot, A. de Juan, R. Tauler, Mcr-als gui 2.0: new features and applications, Chemometrics and Intelligent Laboratory Systems 140 (2015) $1-12$.

[27] L. Yi, N. Dong, Y. Yun, B. Deng, D. Ren, S. Liu, Y. Liang, Chemometric methods in data processing of mass spectrometry-based metabolomics: A review, Analytica chimica acta 914 (2016) 17-34.

[28] T. Azzouz, R. Tauler, Application of multivariate curve resolution alternating least squares (mcr-als) to the quantitative analysis of pharmaceutical and agricultural samples, Talanta 74 (5) (2008) 1201-1210.

[29] B. Debus, D. Kirsanov, V. Panchuk, V. Semenov, A. Legin, Three-point multivariate calibration models by correlation constrained mcr-als: A feasibility study for quantitative analysis of complex mixtures, Talanta 163 (2017) 39-47. 